\title{
Modified arteriosclerosis score predicts the outcomes of diabetic kidney disease
}

Yifan Zhang ${ }^{1,2,3 \dagger}$, Qifeng Jiang ${ }^{4 \dagger}$, Jianteng Xie ${ }^{2,5}$, Chunfang Qi ${ }^{2,5}$, Sheng $\mathrm{Li}^{2,5}$, Yanhui Wang ${ }^{1,2,6}$, Yau Hok Him ${ }^{1,2}$, Zujiao Chen ${ }^{1,2}$, Shaogui Zhang ${ }^{1,2}$, Qiuling Li ${ }^{2,7}$, Yuan Zhu ${ }^{8}$, Ruizhao Li' ${ }^{2}$, Xinling Liang ${ }^{2}$, Xiaoyan Bai ${ }^{2,5}$ and Wenjian Wang ${ }^{1,2,5,7^{*}}$

\begin{abstract}
Background: The significance of renal arteriosclerosis in the prediction of the renal outcomes of diabetic kidney disease (DKD) remains undetermined.

Methods: We enrolled 174 patients with DKD from three centres from January 2010 to July 2017. The severity and extent of arteriosclerosis were analysed on sections based on dual immunohistochemical staining of CD31 and asmooth muscle actin. An X-tile plot was used to determine the optimal cut-off value. The primary endpoint was renal survival (RS), defined as the duration from renal biopsy to end-stage renal disease or death.

Results: The baseline estimated glomerular filtration rate (eGFR) of 135 qualified patients was $45(29 \sim 70) \mathrm{ml} / \mathrm{min}$ per $1.73 \mathrm{~m}^{2}$, and the average $24-\mathrm{h}$ urine protein was $4.52(2.45 \sim 7.66) \mathrm{g} / 24 \mathrm{~h}$. The number of glomeruli in the biopsy specimens was $21.07 \pm 9.7$. The proportion of severe arteriosclerosis in the kidney positively correlated with the Renal Pathology Society glomerular classification $(r=0.28, P<0.012)$, interstitial fibrosis and tubular atrophy (IFTA) $(r=0.39, P<0.001)$, urine protein $(r=0.213, P=0.013)$, systolic BP $(r=0.305, P=0.000)$, and age $(r=0.220, P=$ $0.010)$ and significantly negatively correlated with baseline eGFR $(r=-0.285, P=0.001)$. In the multivariable model, the primary outcomes were significantly correlated with glomerular class (HR: 1.72, Cl: $1.15 \sim 2.57$ ), IFTA (HR: 1.96, Cl: $1.26 \sim 3.06$ ) and the modified arteriosclerosis score (HR: 2.21, Cl: $1.18 \sim 4.13$ ). After risk adjustment, RS was independently associated with the baseline eGFR (HR: 0.97, Cl: $0.96 \sim 0.98$ ), urine proteinuria (HR: 1.10, Cl: $1.04 \sim 1.17$ ) and the modified arteriosclerosis score (HR: 2.01, Cl: 1.10 3.67), and the nomogram exhibited good calibration and acceptable discrimination (C-index $=0.82$, Cl: $0.75 \sim 0.87$ ).
\end{abstract}

Conclusions: The severity and proportion of arteriosclerosis may be helpful prognostic indicators for DKD.

Keywords: Arteriosclerosis, Diabetic kidney disease (DKD), Nomogram, Outcomes, Renal pathology

\footnotetext{
* Correspondence: wwjph@126.com

${ }^{\dagger}$ Yifan Zhang and Qifeng Jiang contributed equally to this work.

${ }^{1} T$ The Second School of Clinical Medicine, Southern Medical University,

Guangzhou 510515, China

2Division of Nephrology, Guangdong Provincial People's Hospital,

Guangdong Academy of Medical Sciences, 106 Zhongshan Er Road, Main

Building, Room 1436, Guangzhou 510080, Guangdong, China

Full list of author information is available at the end of the article
}

(c) The Author(s). 2021 Open Access This article is licensed under a Creative Commons Attribution 4.0 International License, which permits use, sharing, adaptation, distribution and reproduction in any medium or format, as long as you give appropriate credit to the original author(s) and the source, provide a link to the Creative Commons licence, and indicate if changes were made. The images or other third party material in this article are included in the article's Creative Commons licence, unless indicated otherwise in a credit line to the material. If material is not included in the article's Creative Commons licence and your intended use is not permitted by statutory regulation or exceeds the permitted use, you will need to obtain permission directly from the copyright holder. To view a copy of this licence, visit http://creativecommons.org/licenses/by/4.0/. The Creative Commons Public Domain Dedication waiver (http://creativecommons.org/publicdomain/zero/1.0/) applies to the data made available in this article, unless otherwise stated in a credit line to the data. 


\section{Introduction}

Diabetic kidney disease (DKD) is the most common complication in patients exposed to long-term hyperglycaemia [1]. In China, the prevalence of DKD is rising dramatically. Diabetes-related chronic kidney disease (CKD) has overtaken glomerulonephritis as the leading cause of end-stage renal disease (ESRD) [2]. Studies have shown that patients with diabetes in Asia are younger than those in Western countries and are more likely to develop ESRD [3, 4]. However, there are still few biomarkers to predict the progression of DKD to ESRD [5]. Although clinical risk factors, including albuminuria and estimated glomerular filtration rate (eGFR) [6], are most commonly used to predict the progression to ESRD, there is still a lack of pathologic markers, such as those associated with the Oxford classification of IgA nephropathy [7-11], partly because the proportion of patients who receive kidney biopsies remains drastically lower than that of patients with primary glomerulonephritis.

In 2010, the Research Committee of the Renal Pathology Society (RPS) developed a pathologic classification system of diabetic nephropathy [12]. However, the connections of pathological lesions with renal prognosis have not been fully established. An evidence-based approach is needed to better define pathologic lesions within the classification of the DKD spectrum, including the significance of vascular damage. Some studies have suggested that Kimmelstiel-Wilson nodules have a higher risk of ESRD than diffuse DKD [13], while others have found segmental sclerosis and extracapillary hypercellularity to be poor prognostic indicators for ESRD $[14,15]$. Thus, the prognostic significance of pathological parameters in DKD remains controversial [16].

DKD is a common vascular complication of diabetes. Renal ischaemia and endothelial injury play important roles in the pathological processes that promote renal fibrosis and CKD [17-19]. Although the DKD pathologic classification of RPS takes into account and defines renal vascular damage, its prognostic value has not yet been proven $[14,15]$. Because the original arteriosclerosis score for DKD classification was based on the worst artery, this classification only considered the severity of the injured artery but not the proportion of the damaged arteries of all counted arteries.

In the current study, we hypothesized that the proportion of damaged arteries to all arteries in the tissue may contribute to the prognosis of DKD. To validate the significance of renal artery damage in the prediction of renal outcomes of DKD, we employed dual immunoperoxidase staining to better identify arteries with or without lesions and developed a modified arteriosclerosis score for the severity and proportion of all counted arteries. Nomograms were then used to predict the risks of DKD with all clinical characteristics as well as the pathological score [20-22].

\section{Methods \\ Study design and population}

This was a multi-centre, retrospective study of patients with DKD diagnosed by renal pathology. Patients were enrolled between January 2010 and July 2017 from three hospitals (Guangdong Provincial People's Hospital, Wenzhou Central Hospital and Wenzhou People's Hospital). Of the 174 patient-included cohorts, 135 patients were eventually enrolled. The exclusion criteria included patients with DKD coincident with other patterns of kidney injury and inadequate renal tissue samples (renal tissue section should contain at least 10 glomeruli) [12].

All demographic and clinical data were carefully documented from electronic medical clinical records of three hospitals. Demographics included age at the time of biopsy and sex. Clinical parameters collected included body mass index (BMI), blood pressure, hypertension, diabetic retinopathy (DR), diabetes duration, smoking, glycosylated haemoglobin (HbA1c) level, total cholesterol (Chol) level, serum albumin (ALB) level, urine protein (UPRO) level, eGFR, follow-up time, and follow-up serum creatinine $(\mathrm{sCr})$ level. Hypertension was considered in patients with systolic blood pressure greater than $140 \mathrm{mmHg}$, diastolic blood pressure greater than 90 $\mathrm{mmHg}$ or antihypertensive drug administration. DR was defined as the presence of any characteristic lesion as described by the International Clinical Diabetic Retinopathy Disease Severity Scale, which is a grading standard designed according to the Wisconsin Epidemiologic Study of Diabetic Retinopathy (WESDR). Hyperuricaemia was defined as serum uric acid $\geq 420 \mu \mathrm{mol} / \mathrm{L}$ in males or $\geq 360 \mu \mathrm{mol} / \mathrm{L}$ in females. Urinary protein was expressed in $\mathrm{g}$ per $24 \mathrm{~h}$. The eGFR was estimated using the creatinine-based Chronic Kidney Disease Epidemiology Collaboration equation [23].

\section{Outcomes}

The clinical outcomes were considered to evaluate the predictive value of renal pathology variables, ESRD (i.e., haemodialysis, peritoneal dialysis for ESRD, or kidney transplantation), or all-cause death. The follow-up time was defined as the duration from renal biopsy to ESRD or all-cause death [24, 25], the date of last patient contact, or the study end date of July 2017.

\section{Pathology evaluation}

All renal biopsies were performed and processed by standard techniques [24, 25]. The diagnosis of DKD was completed with the findings of light microscopy, immunofluorescence microscopy and electron microscopy as well as supportive clinical information. Any coexisting 
disorders were reviewed to rule out other accompanying glomerular diseases. In addition, dual immunohistochemistry of the paraffin sections with an antibody against $\alpha$-smooth muscle actin ( $\alpha$-SMA, NCL-L-SMA, Aq-meditech, China) and an antibody against CD31 (NCL-L-CD31-607, Aq-meditech, China), which is an endothelial marker, was performed to highlight the arteries.

Glomerular lesions and interstitial lesions were scored according to the DKD pathologic classification issued by RPS $[24,25]$. For vascular lesions of DKD, a modified arteriosclerosis score and modified arteriolar hyalinosis score [26] were employed on dual immunoperoxidase staining sections, which could be more convenient to distinguish arterial damage. In addition, the thickness of the basement membrane under electron microscopy was added as one of the pathological characteristics.

To assess the proportion of all involved arteries distributed in the tissues, severe arteriosclerosis, which was defined as arteries with thickening exceeding the intima thickness, to all arteries in sections was calculated. Since the proportion of severe arteriosclerosis was a continuous variable, $\mathrm{X}$-tile software was used to find the best cut-off value and convert it to hierarchical data [27, 28]. Eventually, the optimal cut-off value for the proportion of severe arteriosclerosis was defined as $50 \%$. We scored extent of arteriosclerosis together as a percentage of the total involved arteries of all severe arteriosclerosis. A score of 0 was assigned when the biopsy specimen showed no arteriosclerosis, a score of 1 was assigned when less than $50 \%$ severe arteriosclerosis was present, and a score of 2 was assigned when more than $50 \%$ severe arteriosclerosis was present. RPS glomerular class, IFTA, interstitial inflammation, arteriolar hyalinosis score, and arteriolar hyalinosis score were determined following standard procedures [24, 25].

Without knowledge of the clinical outcomes, two experienced nephropathologists (Qifeng Jiang and Yifan Zhang) were assigned to independently evaluate and score light microscopy slides. Pathological scoring disagreements were resolved by consensus.

\section{Statistical analyses}

Variables with a normal distribution are presented as the means and standard deviations and were subjected to unpaired Student's $t$-test between groups, one-way analysis of variance (ANOVA) in multiple groups, or Pearson's test for correlation analysis. Nonparametric variables are presented as the medians and interquartile ranges (IQR) and were analysed with the Mann-Whitney test between groups, Kruskal-Wallis test in multiple groups, or Spearman rank correlation test. Categorical variables were expressed as percentages and compared using the Pearson $\mathrm{X}^{2}$ test.
The modified arteriosclerosis score cut-off value was analysed using the X-tile plot [28]. Survival analysis for renal survival or death was performed using KaplanMeier (KM) survival curves with log-rank tests and the Cox proportional hazards model to identify the potential association between clinical and pathologic variables and outcomes. Variables with a significance of $P<0.1$ in the univariate models were included in the multivariable models [29]. Three multivariable models were established, and the first multivariable model (the "Clinical Model") included only those clinical variables significant to $P<0.1$ in the univariate models. The second multivariable model (the "Pathological Model") included only those pathological variables significant to $P<0.1$ in the univariate models. The "Fully Risk-Adjustment Model" incorporated clinical variables significant at $P<0.05$ in the clinical model, as well as pathological variables significant at $P<0.05$ in the pathological model. Then, the AUCs of different risk variables and two models in predicting RS were estimated using time-dependent receiver operating curves (ROCs).

To estimate median and individual postdiagnosis renal survival probabilities at 1,3 , and 5 years, a nomogram was constructed based on the "Fully Risk-Adjustment Model" [20]. Validation of the prediction model was performed using two parameters: discrimination and calibration [30]. Discrimination was measured by the concordance index (C-index), whereas calibration was evaluated using a calibration plot. By convention, a concordance index of less than 0.6 indicates poor discrimination, $\quad 0.60-0.75$ indicates possibly helpful discrimination, and more than 0.75 indicates outstanding discrimination [30].

All tests used were two-sided, with a $P$-value $<0.05$ considered statistically significant. All statistical analyses were performed using X-tile software version 3.6.1 (http://tissuearray.org), GraphPad Prism 7 software (GraphPad Software) or R version 3.6.3 (Foundation for Statistical Computing, Vienna, Austria; http://www.Rproject.org/).

\section{Results}

Clinical characteristics at presentation and during followup

Of 174 patients with biopsy-proven DKD, 37 patients were excluded due to coincidence with other kidney diseases, and 2 patients were excluded due to inadequate tissue samples. Finally, 135 patients were enrolled in this study. The mean number of glomeruli for each biopsy specimen was $21.07 \pm 9.7$. During the 21 -month followup (interquartile range, 15-38 months) [31], 10 patients (7.4\%) lost connection, 57 patients (42.2\%) developed ESRD, 5 individuals (3.7\%) died before reaching ESRD, 
and 9 individuals (6.7\%) died after progression to ESRD. The flowchart of the study is shown in Fig. 1.

The baseline clinical data of 135 DKD patients are listed in Table 1 . The average age of the enrolled participants at baseline was $52.13 \pm 10.42$ years, and the majority of patients were male $(71.9 \%)$, with a BMI of $24.74 \pm$ $3.88 \mathrm{~kg} / \mathrm{m}^{2}$. The median duration of diabetes was 7 (311) years, with HbA1c of $7.72 \pm 3.88 \%$, SBP of $157.12 \pm$ $25.92 \mathrm{mmHg}$, and total cholesterol of 5.89 (4.9-6.81) $\mathrm{mmol} / \mathrm{L}$. The median urine protein at enrolment was $4.52(2.45-7.66) \mathrm{g} / 24 \mathrm{~h}$, with a median baseline eGFR of $45(29-70) \mathrm{ml} / \mathrm{min}$ per $1.73 \mathrm{~m}^{2}$.

The pathological characteristics of the patients are tabulated in Table 2.
As depicted in Fig. 2, the proportion of severe arteriosclerotic lesions exhibited a correlation with glomerular classification $(r=0.28, P<0.012)$ and IFTA $(r=0.39, P<$ 0.001 ) (Fig. $3 \mathrm{a}$ and b). In glomerular class IV, the proportion of severe arteriosclerosis was higher than in other classes, but there was no significant difference between class IIb and class III. In some cases, the severity of arteriosclerosis of class III was not more severe than that of class IIb (Figs. 2 and 3a). IFTA is more closely related to arteriosclerosis, and a continuous increase in progressive fibrosis parallels the increase in the proportion of severe arteriosclerosis (Fig. $3 a$ and b).

Figure 4 shows the correlation between the proportion of severe atherosclerosis and clinical variables. The

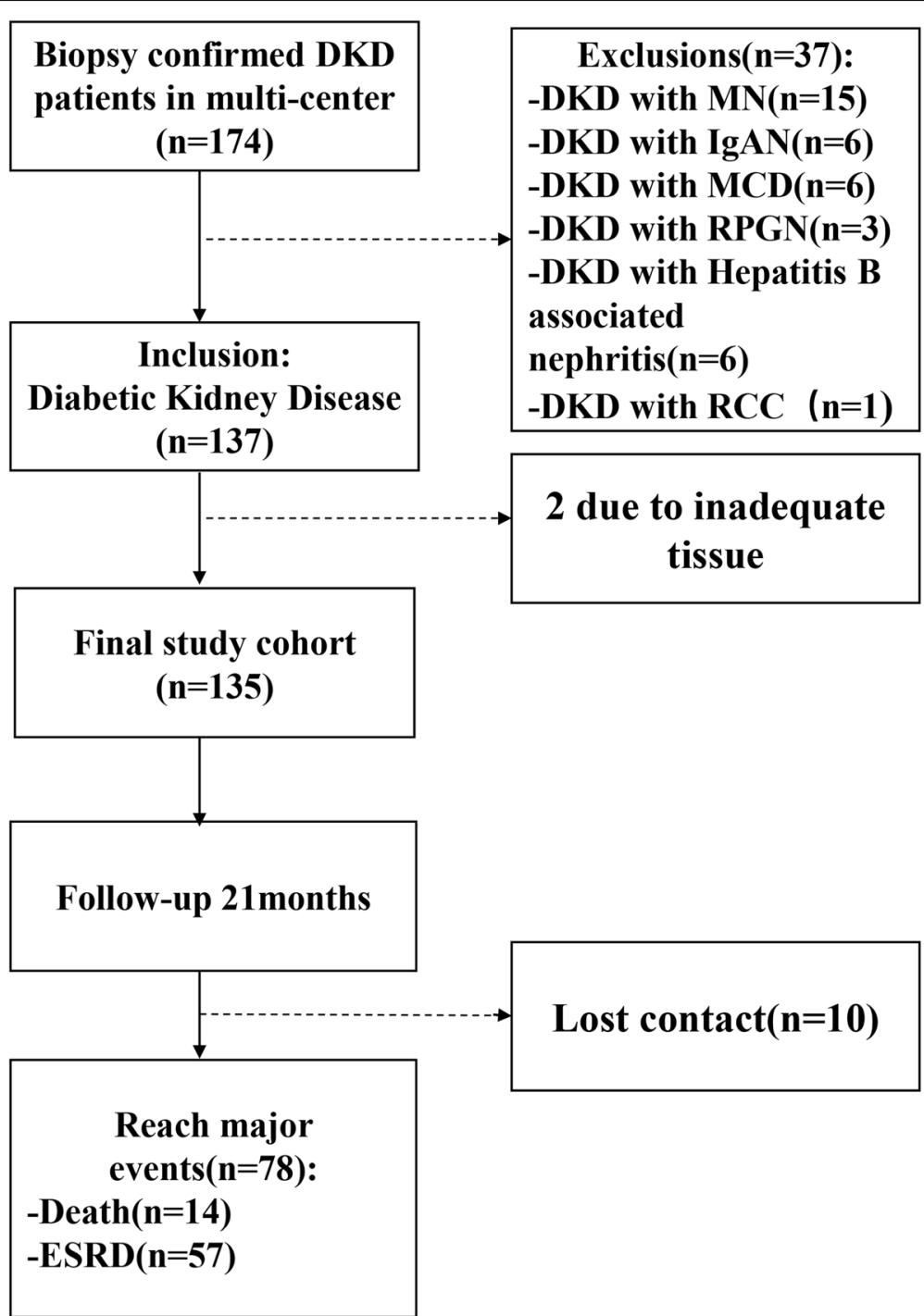

Fig. 1 Flow diagram of the enrolment process. Abbreviations: DKD, diabetic kidney disease; ESRD, end-stage renal disease; IgAN, IgA nephropathy; MCD, minimal change disease; MN, membranous nephropathy; RCC, renal cell carcinoma; RPGN, rapidly progressive glomerulonephritis 
Table 1 Clinical characteristics at the time of biopsy in 135 patients with DKD

\begin{tabular}{|c|c|}
\hline Characteristics & Results \\
\hline Age (years) & $52.13 \pm 10.42$ \\
\hline Male sex & $71.9 \%$ \\
\hline Smoke & $32.6 \%$ \\
\hline Hypertension & $93.3 \%$ \\
\hline Hyperuricemia & $42.2 \%$ \\
\hline Diabetic retinopathy & $65.9 \%$ \\
\hline Diabetes duration (years) & $7(3-11)$ \\
\hline BMI $\left(\mathrm{kg} / \mathrm{m}^{2}\right)$ & $24.74 \pm 3.88$ \\
\hline Systolic BP (mmHg) & $157.12 \pm 25.92$ \\
\hline HbA1c (\%) & $7.72 \pm 1.78$ \\
\hline Total Cholesterol (mmol/L) & $5.89(4.9-6.81)$ \\
\hline Albumin $(g / L)$ & $27.67 \pm 7.13$ \\
\hline Urine protein $(g / 24 \mathrm{~h})$ & $4.52(2.45-7.66)$ \\
\hline Baseline eGFR (ml/min per $\left.1.73 \mathrm{~m}^{2}\right)$ & $45(29-70)$ \\
\hline
\end{tabular}

Data are expressed as the means $\pm S D$, medians (interquartile range), or percentages

Abbreviations: BMI body mass index, DKD diabetic kidney disease, eGFR estimated glomerular filtration rate, HbA1c glycosylated haemoglobin

Table 2 Pathologic scoring of kidney biopsy specimens from 135 patients with DKD

\begin{tabular}{|c|c|c|}
\hline Pathologic scores & Pathologic definitions & Prevalence, $\mathrm{n}(\%)$ \\
\hline \multicolumn{3}{|c|}{ RPS Glomerular class } \\
\hline 1 & Mild & $1(0.7 \%)$ \\
\hline Ila & Mild mesangial expansion in $>25 \%$ of mesangium & $15(10.2 \%)$ \\
\hline$\| \mathrm{b}$ & Severe mesangial expansion in $>25 \%$ of mesangium & $17(11.6 \%)$ \\
\hline III & At least one KW lesion & $86(58.5 \%)$ \\
\hline IV & Advanced diabetic glomerulosclerosis & $16(10.9 \%)$ \\
\hline \multicolumn{3}{|l|}{ IFTA } \\
\hline 0 & None & $0(0 \%)$ \\
\hline 1 & $1-25 \%$ & $25(18.5 \%)$ \\
\hline 2 & $26-50 \%$ & $45(33.3 \%)$ \\
\hline 3 & $>50$ & $65(48.2 \%)$ \\
\hline \multicolumn{3}{|c|}{ Interstitial inflammation } \\
\hline 0 & None & $0(0 \%)$ \\
\hline 1 & Infiltration only in relation to IFTA & $88(65.2 \%)$ \\
\hline 2 & Infiltration in areas without IFTA & $47(34.8 \%)$ \\
\hline \multicolumn{3}{|c|}{ Arteriolar hyalinosis score } \\
\hline 0 & Absent & $3(2.2 \%)$ \\
\hline 1 & At least one area of arteriolar hyalinosis & $12(8.9 \%)$ \\
\hline 2 & More than one area of arteriolar hyalinosis & 120(88.9) \\
\hline \multicolumn{3}{|c|}{ Modified arteriolar hyalinosis score } \\
\hline 0 & Absent & $3(2.2 \%)$ \\
\hline 1 & Mild-to-moderate PAS-positive hyaline thickening in at least one arteriole & $12(8.9 \%)$ \\
\hline 2 & moderate-to-severe PAS-positive hyaline thickening in more than one arteriole & $49(36.3 \%)$ \\
\hline 3 & Severe PAS-positive hyaline thickening in many arterioles & $71(52.6 \%)$ \\
\hline \multicolumn{3}{|c|}{ Arteriosclerosis score ${ }^{a}$} \\
\hline 0 & No intimal thickening & $0(0 \%)$ \\
\hline 1 & Intimal thickening less than thickness of media & $63(46.7 \%)$ \\
\hline 2 & Intimal thickening greater than thickness of media & $72(53.3 \%)$ \\
\hline \multicolumn{3}{|c|}{ Modified arteriosclerosis score ${ }^{b}$} \\
\hline 1 & 0-49\% The Proportion of arteries with intima thickness greater than media thickness in the biopsy & 113(83.7\%) \\
\hline 2 & $\geq 50 \%$ & $22(16.3 \%)$ \\
\hline
\end{tabular}

Abbreviations: IFTA interstitial fibrosis and tubular atrophy, RPS the Renal Pathology Society

a original arteriosclerosis score the most severely affected artery in the biopsy; ${ }^{\text {b }}$ Modified arteriosclerosis score the proportion of severe arteriosclerosis in the biopsy 


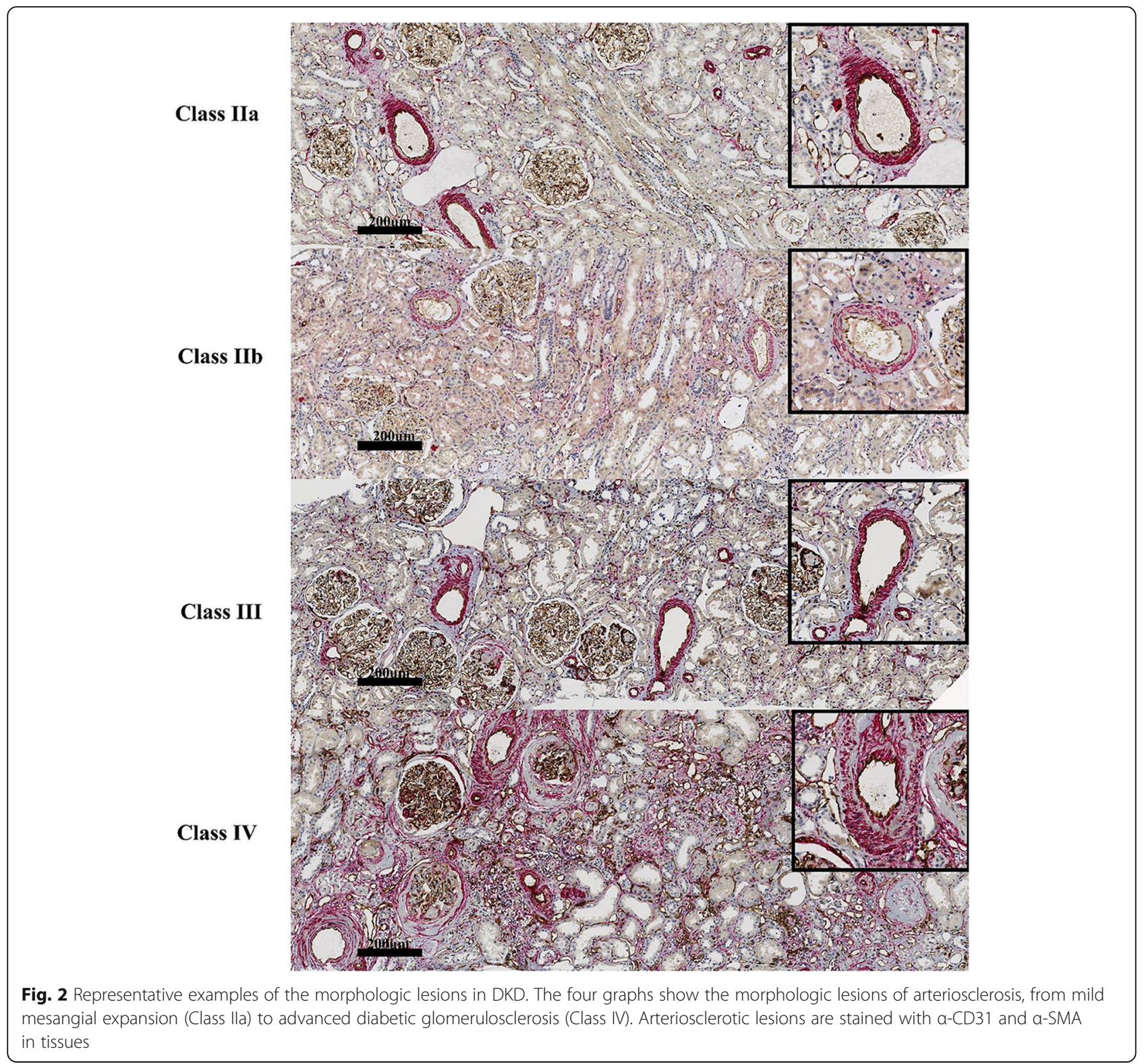

proportion of severe atherosclerosis was significantly negatively correlated with baseline eGFR $(r=-0.285$, $P=0.001$ ) but significantly positively correlated with urine protein $(r=0.213, P=0.013)$, SBP $(r=0.305, P=$ $0.000)$, and age $(r=0.221, P=0.010)$. Univariable and multivariable Cox regression analyses were used to explore the association between clinical characteristics and renal survival (ESRD or death). Only clinical variables that showed significance in the univariate analysis $(P<$ 0.1 ) were included in the multivariable clinical model. The only clinical variables to remain significant in the multivariable clinical model were baseline eGFR, urine proteinuria and albumin (Table 3 ).
As shown in Table 4, univariate analysis of pathological factors revealed that RPS glomerular class (hazard ratio, HR: 2.24, 95\% confidence interval, CI: 1.47-3.40), IFTA (HR: 2.44, CI: 1.61-3.69), arteriosclerosis score (HR: 2.14, CI: 1.26-3.63) and modified arteriosclerosis score (HR: 4.15, CI: 2.35-7.36) were significantly associated with renal survival. KM survival curve for renal survival was performed. Higher RPS glomerular class (Fig. 5a, $P=0.00026$ ), IFTA (Fig. 5b, $P<0.0001$ ), arteriosclerosis score (Fig. 5 c, $P$ $=0.0035)$ and modified arteriosclerosis score (Fig. $5 \mathrm{~d}$, $P<0.0001)$ were correlated with shorter RS time in DKD patients. 


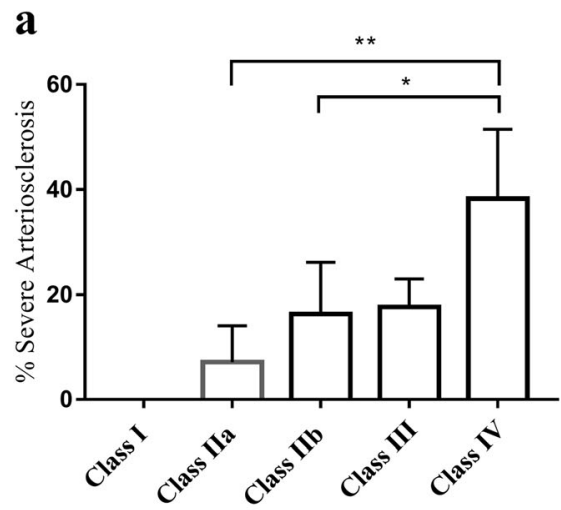

Glomerular classification

b

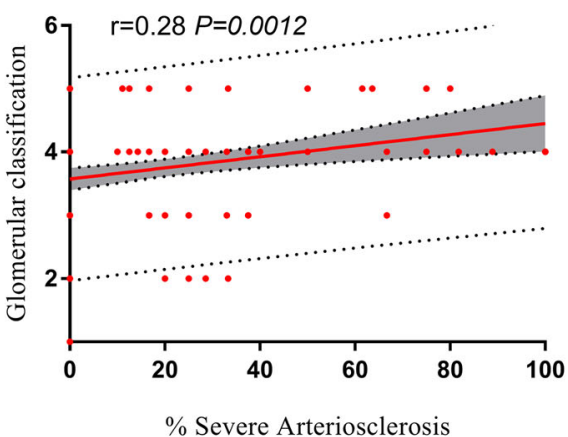

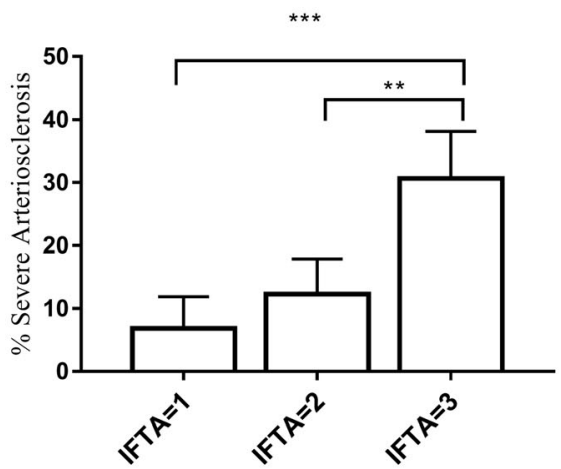

Interstital lesion

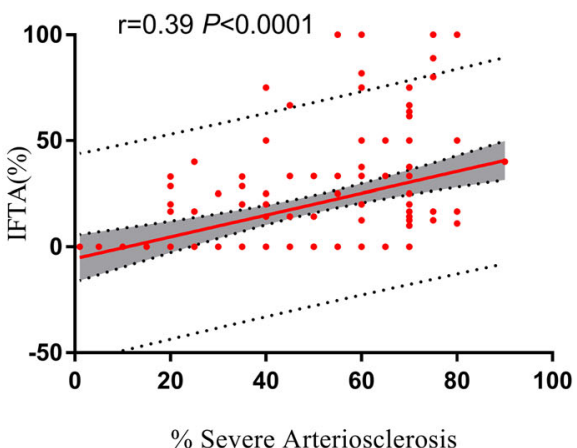

Fig. 3 Relationship between vascular damage and glomerular class and IFTA in diabetic nephropathy. a Histogram shows the proportion of severe arteriosclerosis changes with glomerular classification and IFTA progression. Figure (b) Scatter plot showing the relationship between the proportion of severe arteriosclerosis and glomerular classification and IFTA. Abbreviations: IFTA, interstitial fibrosis and tubular atrophy

Pathological variables with $P<0.05$ in univariate analysis were also used to construct two multivariable models. In the pathological model, only RPS glomerular class and IFTA were statistically significant if RPS glomerular class (HR: 1.72, CI: 1.153-2.57), IFTA (HR: 1.96, CI: 1.26-3.06) or arteriosclerosis scores (HR: 1.29, CI: $0.73-2.29)$ were included. However, if the modified arteriosclerosis score (HR: 2.21, CI: 1.18-4.13) was used instead of the arteriosclerosis score in the model, all three variables were statistically significant. In the second multivariable model, clinical features (baseline eGFR, urine proteinuria and albumin) and RPS glomerular class, IFTA or modified arteriosclerosis score were included. The full risk-adjustment model showed that only baseline eGFR (HR: 0.97, CI: 0.96-0.98), urine proteinuria (HR: 1.10, CI: 1.04-1.17) and modified arteriosclerosis score (HR: 2.01, CI: 1.10-3.67) were independently associated with RS. The forest plot in Fig. 6 shows the results of the stepwise model selection for time to renal survival using multivariable Cox regression.
Figures S1 and S2 show the AUCs of different risk variables and two models (pathological model and full riskadjustment model) for RS at 24 months after the start of follow-up using time-dependent ROC analysis. In the comparison of individual factors, eGFR showed the highest correlation with RS, with an AUC of 0.81 (95\% CI: 0.71-0.92). The full risk-adjustment model had stronger predictive power than the pathological model after correction for multiple factors.

A prognostic nomogram for 1-, 3-, and 5-year RS was established (Fig. 7a). Baseline eGFR, urine proteinuria and modified arteriosclerosis score, which were shown to be independent predictors for renal survival in the multivariable Cox regression analysis, were included in the nomogram.

In terms of discrimination, the C-index for renal survival was 0.82 (95\% CI: 0.75-0.87). The calibration curve for the probability of survival at 1, 3 or 5 years after biopsy showed optimal agreement between the prediction by the nomogram and the actual observation (Fig. 7b-d). According to the calibration curve, the predicted survival 
a

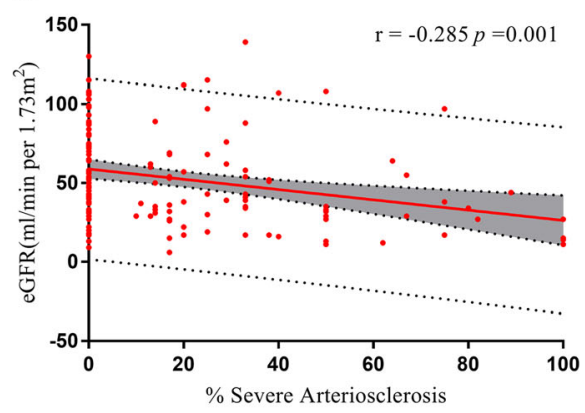

c

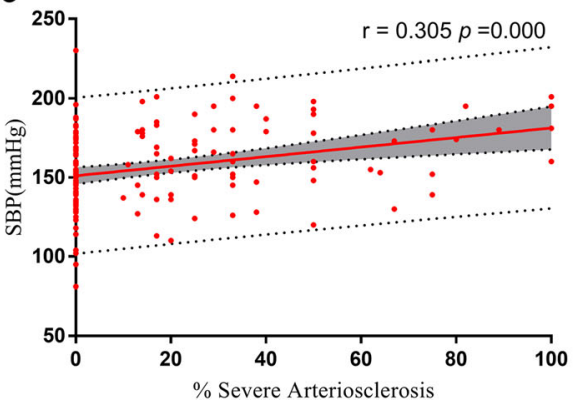

b

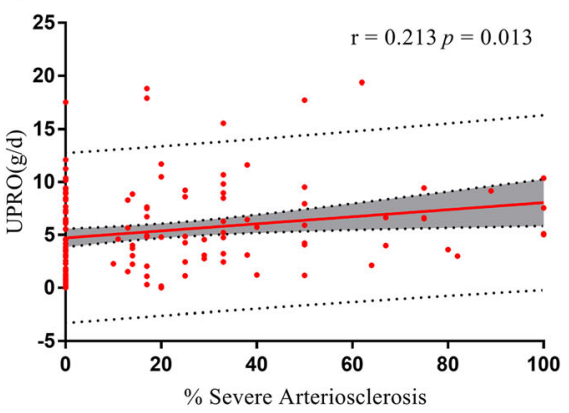

d

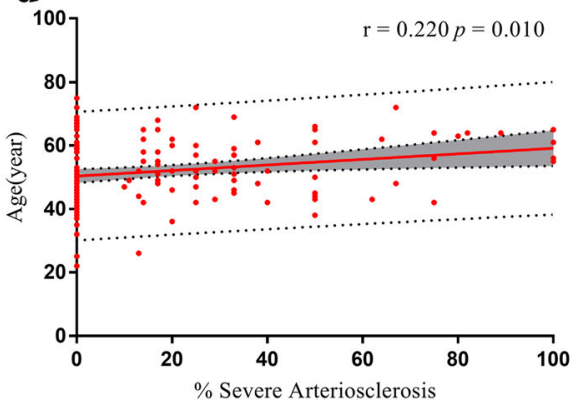

Fig. 4 Correlation between the proportion of severe atherosclerosis and clinical variables. a The correlation between the proportion of severe atherosclerosis and baseline eGFR. $\mathbf{b}$ The correlation between the proportion of severe atherosclerosis and UPRO. $\mathbf{c}$ The correlation between the proportion of severe atherosclerosis and SBP. $\mathbf{d}$ The correlation between the proportion of severe atherosclerosis and age. All significant correlations are given as Pearson's $r, P<0.05$. The grey area represents the $95 \%$ confidence interval. Abbreviations: eGFR, estimated glomerular filtration rate; SBP, systolic blood pressure; UPRO, urine protein

Table 3 Correlations between clinical features and outcome (endpoint:ESRD or death)

\begin{tabular}{|c|c|c|c|c|}
\hline Characteristics & Univariate Models HR $(95 \% \mathrm{Cl})$ & $P$ Value & Clinical Model HR $(95 \% \mathrm{Cl})$ & $P$ Value \\
\hline Age & $0.99(0.97-1.02)$ & 0.79 & & \\
\hline Female sex & $1.24(0.74-2.07)$ & 0.41 & & \\
\hline Smoke & $0.88(0.50-1.56)$ & 0.66 & & \\
\hline Diabetic retinopathy & $1.12(0.66-1.91)$ & 0.67 & & \\
\hline Diabetes duration & $0.98(0.94-1.03)$ & 0.41 & & \\
\hline BMI & $1.00(0.93-1.07)$ & 0.99 & & \\
\hline Systolic BP & $1.02(1.01-1.03)$ & 0.00 & & \\
\hline $\mathrm{HbA1c}$ & $0.87(0.73-1.02)$ & 0.08 & & \\
\hline Total Cholesterol & $1.17(1.05-1.31)$ & 0.00 & & \\
\hline Alb & $0.94(0.90-0.97)$ & 0.00 & $0.95(0.90-0.99)$ & 0.01 \\
\hline Urine protein & $1.15(1.08-1.21)$ & 0.00 & $1.08(1.01-1.15)$ & 0.03 \\
\hline Baseline eGFR & $0.96(0.95-0.98)$ & 0.00 & $0.96(0.95-0.98)$ & 0.00 \\
\hline
\end{tabular}

Clinical Model: Clinical variables with significance at $P<0.1$ in univariate models (baseline eGFR, proteinuria, albumin, systolic BP, HbA1c, total cholesterol) were included in the multivariable clinical model, and those that remained significant in the multivariable clinical model were baseline eGFR, urine proteinuria and albumin

Abbreviations: Alb albumin, BMI body mass index, $\mathrm{Cl}$ confidence interval, eGFR estimated glomerular filtration rate, ESRD end-stage renal disease, HbA1c glycosylated haemoglobin 
Table 4 Univariate and multivariable models for time to outcome (endpoint:ESRD or death)

\begin{tabular}{|c|c|c|c|c|c|c|}
\hline Characteristics & $\begin{array}{l}\text { Univariate Model } \\
\text { HR }(95 \% \mathrm{Cl})\end{array}$ & $P$ Value & $\begin{array}{l}\text { Pathological } \\
\text { Model HR }(95 \% \mathrm{Cl})\end{array}$ & $P$ Value & $\begin{array}{l}\text { Fully Risk-Adjustment } \\
\text { Model HR }(95 \% \mathrm{Cl})\end{array}$ & $P$ Value \\
\hline \multicolumn{7}{|l|}{ Clinical features } \\
\hline \multicolumn{7}{|l|}{ Alb } \\
\hline Baseline Urine protein & & & & & $1.10(1.04-1.16)$ & 0.00 \\
\hline Baseline eGFR & & & & & $0.97(0.95-0.98)$ & 0.00 \\
\hline \multicolumn{7}{|l|}{ Pathological features } \\
\hline RPS Glomerular class & $2.24(1.47-3.40)$ & 0.00 & $1.68(1.13-2.50)$ & 0.01 & & \\
\hline IFTA & $2.44(1.61-3.69)$ & 0.00 & $1.83(1.18-2.84)$ & 0.01 & & \\
\hline Interstitial inflammation & $1.59(0.96-2.62)$ & 0.07 & & & & \\
\hline Arteriosclerosis score & $2.14(1.26-3.63)$ & 0.01 & & & & \\
\hline Modified Arteriosclerosis score & $4.15(2.35-7.36)$ & 0.00 & $1.44(1.04-1.99)$ & 0.03 & & \\
\hline Arteriolar hyalinosis score & $2.43(0.81-7.34)$ & 0.12 & & & & \\
\hline Modified Arteriolar hyalinosis score & $1.15(0.79-1.67)$ & 0.46 & & & & \\
\hline GBM thickness & $1.00(0.99-1.00)$ & 0.86 & & & & \\
\hline
\end{tabular}

Pathological model: RPS, IFTA and Modified arteriosclerosis score; The fully risk-adjustment model: clinical features (eGFR, proteinuria, albumin) and RPS class, IFTA, Modified arteriosclerosis score

Abbreviations: Alb albumin, $C l$ confidence interval, eGFR estimated glomerular filtration rate, ESRD end-stage renal disease, GBM glomerular basement membrane, IFTA interstitial fibrosis and tubular atrophy, RPS the Renal Pathology Society

probabilities at 1,3 and 5 years were in line with the actual survival probability, and the confidence interval intersected with the $45^{\circ}$ diagonal. In addition, the confidence interval of some prediction probabilities was very wide, which indicated that the sample size needs to be increased for further assessment.

\section{Discussion}

Herein is a detailed study of the association of advanced vascular lesions and clinical outcomes in patients with biopsy-proven DKD. With respect to the first research question, we found that the proportion of severe arteriosclerosis was an independent predictor of clinical progression to ESRD for DKD patients. In lupus nephritis and IgA nephropathy, some studies have supported vascular lesions as independent risk factors for predicting renal disease progression [32-35]. Although the pathological classification of DKD included the vascular score in the original literature, the authors did not validate the relationship between vascular lesions and renal prognosis [12]. Furthermore, we found that studies implementing specific analyses based on the correlation of renal vascular lesions and renal prognosis were scarce.

Yu An et al. [36] studied more than 300 patients with type 2 DKD confirmed by biopsy and found that vascular lesion scores demonstrated no association with renal outcomes. However, a different study demonstrated that the presence of arteriolar hyalinosis and arteriosclerosis were associated with renal replacement therapy (RRT) initiation in univariate analyses, although there was no statistical significance in multivariable analyses [37]. The original classification of arteriosclerosis in DKD only assessed the most severe arteriosclerosis but did not assess the proportion of the arteries involved. This may have caused sampling error, and we therefore revised the new scoring systems for vascular lesions to highlight the extent of involvement throughout the tissue. In our study, we found that the higher the proportion of severe arteriosclerosis was, the poorer the baseline renal function, and the more rapidly the creatinine level progressed in the future. Similar to previous research on lupus nephritis, we also found that the proportion of arteriosclerosis was associated with proteinuria. Therefore, we established a new arteriosclerosis score based on the proportion of severe arteriosclerosis.

In the present study, the original arteriosclerosis score was a risk factor in univariate analysis but not in multivariable analysis (Fig. 5c, Table 4). However, univariate and multivariable analyses demonstrated that the modified arteriosclerosis score was an independent factor for prognosis in DKD (Fig. 5d, Fig. 6, and Table 4). Compared with the previous scoring system, we believe the new scoring system can distinguish DKD, which is more likely to worsen. Bohle et al. [38] described that increases in vascular disease correlated with more severe glomerular disease. Gambara et al. [39] also described different renal patterns in diabetic patients that led to renal dysfunction, a subset of which was due to significant vascular sclerosis. Salvatore et al. [40] proposed that the spectrum of ischaemic glomerular disease and podocyte injury may depend on 


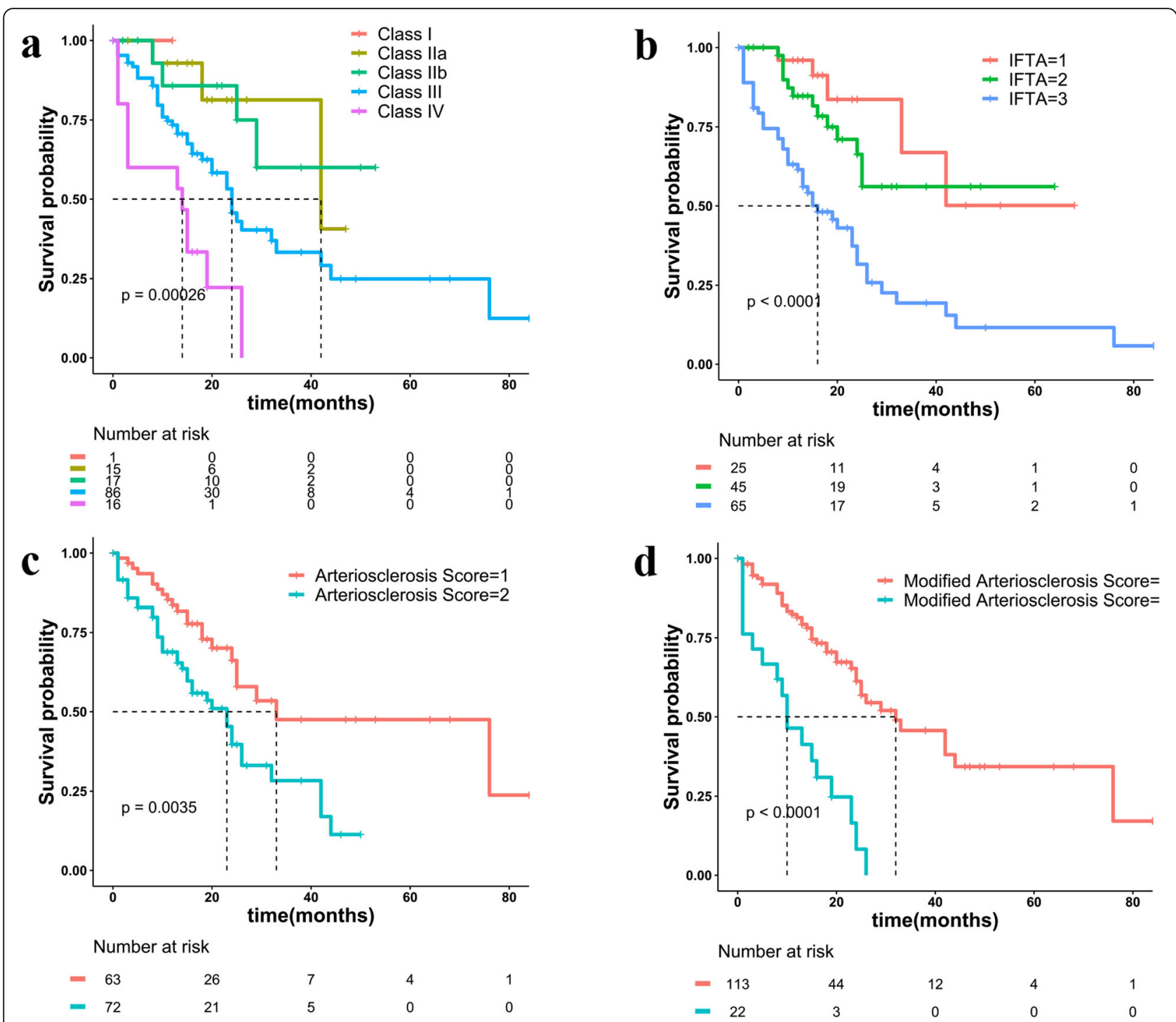

Fig. 5 Kaplan-Meier curves of RS according to pathologic variables. a Glomerular classification of DN; $\mathbf{b}$ IFTA; c arteriosclerosis score; $\mathbf{d}$ modified arteriosclerosis score

the degree and duration of micro- and macrovascular occlusion and that severe vascular changes in diabetic nephropathy lead to hypoxic injury.

Arteriolar hyalinosis is another indicator of vascular lesions. We also modified this scoring system; however, neither the original indicator nor the improved indicator was statistically significant in our study. This result is controversial, however, because some studies have suggested that, similar to the role of hyaline arteriolosclerosis in renal transplantation [41, 42], this pathological feature is associated with the progression of diabetic kidney disease [40]. This phenomenon might be associated with the prevalence of severe arteriolar hyalinosis in our DKD cohort.
More than one vessel of moderate to severe arteriolar hyalinosis was commonly observed in $88.9 \%$ of patients with diabetic nephropathy, suggesting that this index in the classification was incapable of discriminating lesions with various severities for our cohort. This result was similar to a previous study [36].

Glomerular lesions are the most characteristic pathologic changes of DKD. Although the glomerular classification of RPS aims to help reveal the progression of DKD, its prognostic value remains to be verified. In their recent study on patients with biopsy confirmed DKD, Mottl et al. [14] found that glomerular pathologic classification had statistical significance for predicting renal prognosis only in the single-factor 


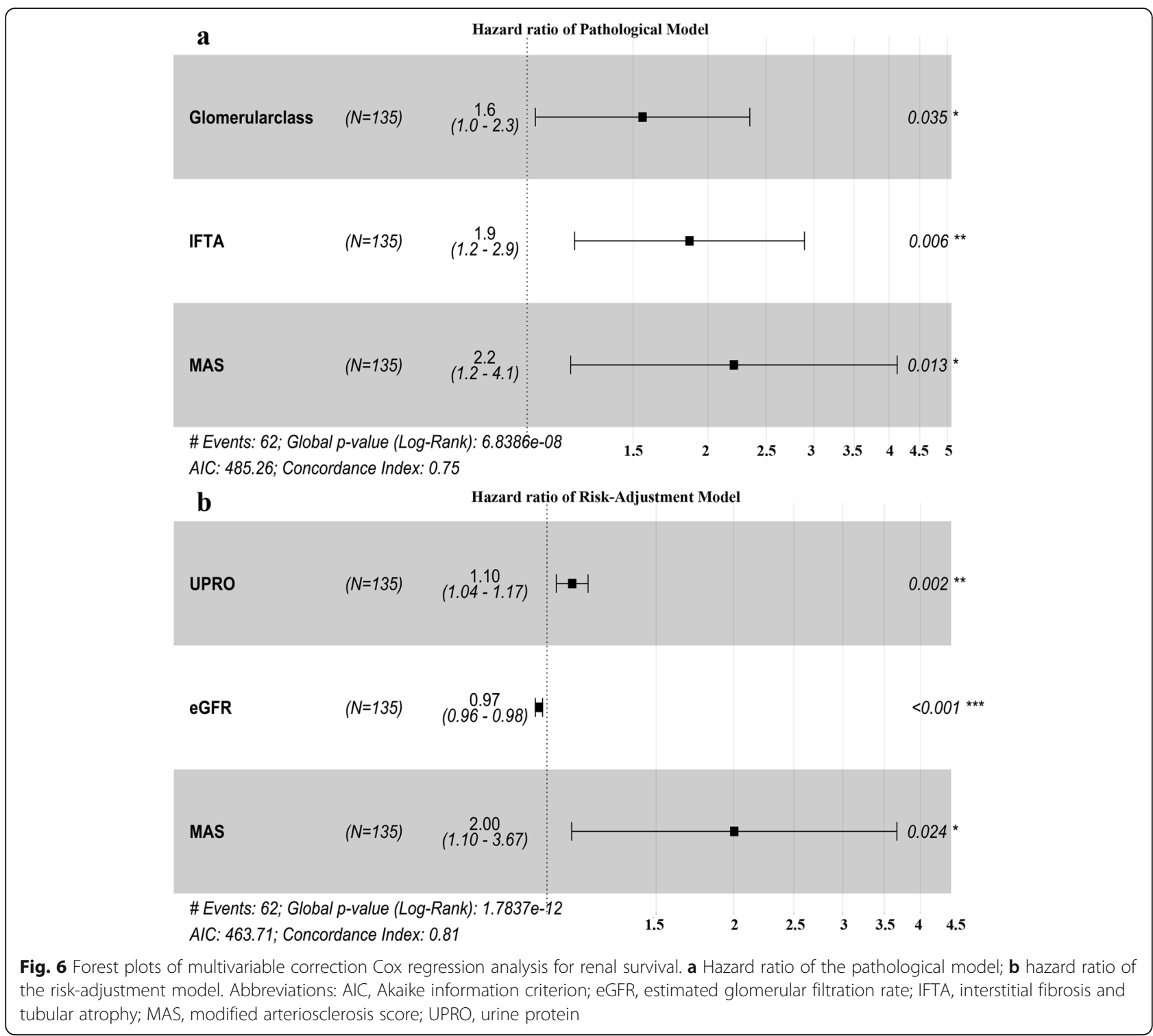

analysis; however, it was not statistically significant in the multifactor analysis. Two other research teams also reported that the renal prognostic significance of the glomerular pathologic classification lost power after multivariate correction $[16,43]$. In contrast, several studies have shown a significant correlation between the RPS DKD classification and decreased GFR after multivariate correction [36, 44-46]. With regard to tubulointerstitial lesions, studies conducted by An Yu et al. [36] and Mise et al. [46] suggested that the IFTA score is an independent risk factor for renal prognosis of diabetic nephropathy, but the research conducted by Mottl et al. [14] suggested that IFTA has no statistical significance for renal prognosis of DKD. In our study, glomerular lesions and IFTA were statistically significant in both univariate and pathological models but not after adjustment for proteinuria, eGFR, serum albumin levels, or arteriosclerosis.

On the basis of previous work, we established a novel user-friendly renal survival prediction model built on three key parameters (urine protein, eGFR and modified arteriosclerosis score) and provided a prognostic nomogram and score [20]. As a tool to predict clinical prognosis, nomograms are widely used in oncology and other medical aspects to help clinical decision-making. Nomograms satisfied our need for an integrated biological and clinical model to realize the goal of personalized medicine; thus, it is reasonable to predict renal prognosis of DKD with a nomogram. The present work revealed considerable heterogeneity among DKD patients regarding their renal survival risk profiles. Our nomogram could provide the nephrologist with a precise probability of 


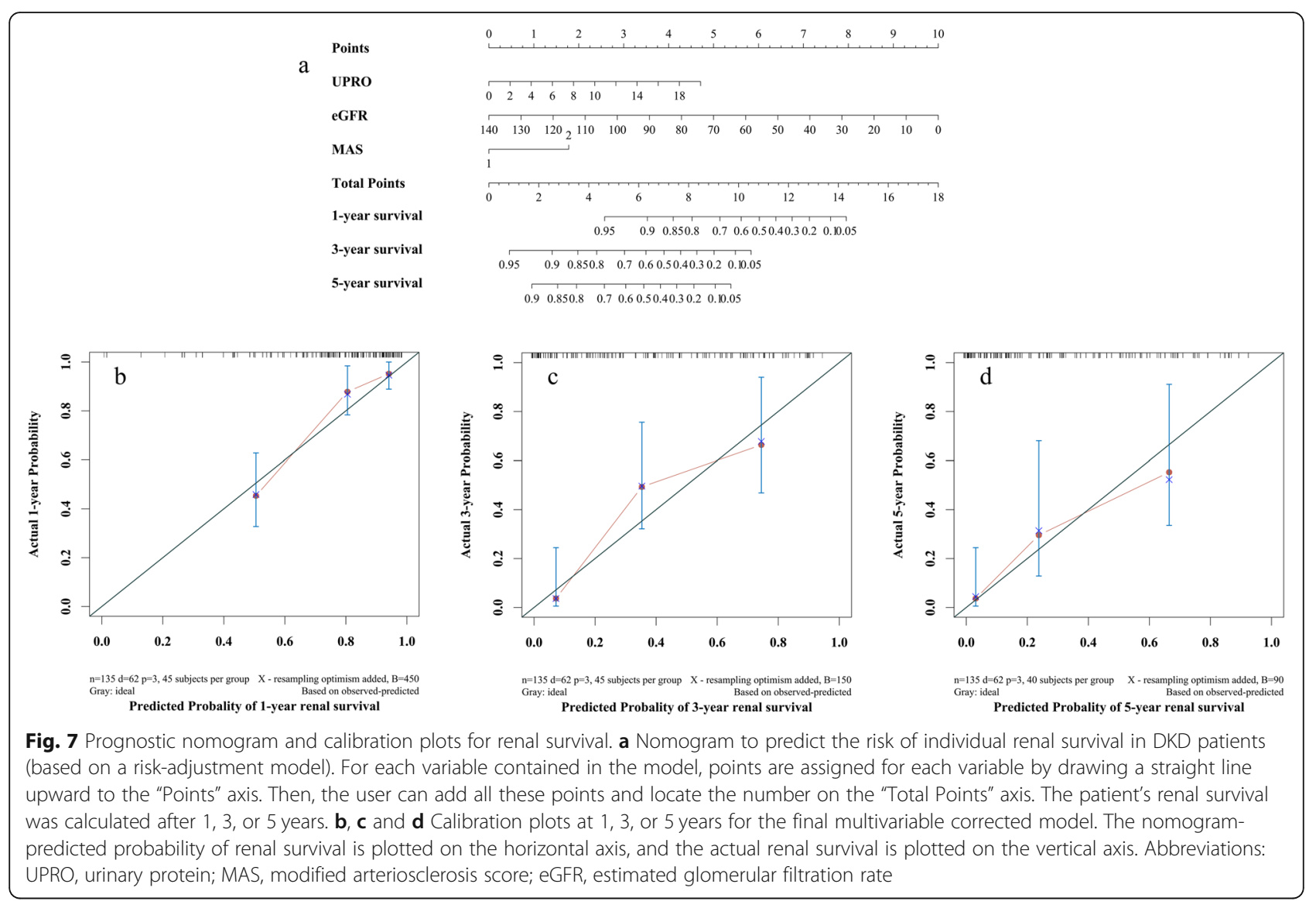

renal survival in patients with DKD and might offer an opportunity to define risk-adapted strategies for DKD management in the future.

Our study has multiple strengths. The main strength of our study is that we illustrated the modified arteriosclerosis score vascular lesion as an independent marker of time to diabetic ESRD with a modified definition. Our method could identify DKD patients with a worse prognosis. Furthermore, the new immunohistochemical method we performed showed more comprehensive characteristics of vascular lesions than conventional staining, which may be used as a reference for the pathological score of DKD in the future. Third, our study was constructed in the framework of a multicentre cohort study with a broad spectrum of parameters available at diagnosis. Multicentre studies can better represent real-world heterogeneity and are more generalizable than single-centre studies. Moreover, we created a nomogram for DKD patients to help nephrologists make clinical decisions. Nomograms have the ability to generate an individual probability of a clinical event by integrating diverse prognostic and determinant variables, which meets our desire for biologically and clinically integrated models and fulfils our drive towards personalized medicine. Finally, the blinded evaluation of renal biopsy is another strength of this study.

Despite these strengths, some limitations must be considered when interpreting our findings. First, it was a retrospective study. However, all end points were based on objectively measured laboratory values that reduced the chance for bias. Second, it was a small sample size. Therefore, our findings are hypothetical and need to be reexamined with a larger data set. Third, we did not evaluate the therapeutic interventions during follow-up, which may have had miscellaneous impacts on renal prognosis. Finally, histologic changes were assessed based on a simple classification, but diabetic glomerulopathy is far more complicated.

In conclusion, we present here a novel renal survival prediction model based on three key independent prognostic factors. Other studies are required to determine whether the modified arteriosclerosis score can be applied as a novel, independent marker of time to ESRD for DKD patients. The prognostic nomogram and the score proposed may help offer the opportunity to define risk-adapted strategies for DKD management in the future. 


\section{Supplementary Information}

The online version contains supplementary material available at https://doi. org/10.1186/s12882-021-02492-x.

Additional file 1: Figure S1. Time-dependent ROC of different risk factors for RS at 24 months of follow-up. Abbreviations: AUC, area under curve; $\mathrm{Cl}$, confidence interval; eGFR, estimated glomerular filtration rate; IFTA, interstitial fibrosis and tubular atrophy; MAS, modified arteriosclerosis score; UPRO, urine protein.

Additional file 2: Figure S2. Time-dependent ROC of different models for RS at 24 months of follow-up.

\section{Acknowledgements}

None declared.

\section{Authors' contributions}

We declare that all the listed authors have participated actively in the study and that all meet the requirements of authorship. Wenjian Wang, Yifan Zhang and Xinling Liang contributed to the conception and design of the work. Jianteng Xie, Sheng Li, Yanhui Wang, Yuan Zhu, Ruizhao Li and Zujiao Chen contributed to the acquisition of data. YAU HOK HIM, Chunfang Qi, Shaogui Zhang and Qiuling Li contributed to the analysis and interpretation of the data. Yifan Zhang and Qifeng Jiang wrote the draft of the manuscript. Wenjian Wang revised the manuscript critically for important intellectual content. All authors have read and approved the manuscript.

\section{Funding}

This work was supported by grants from the National Natural Science Foundation (NO. 81270816 \& NO. 81470974 to W.J.W.) and High-level Hospital Construction Project of Guangdong Province (DFJH201908 to W.J.W.)

\section{Availability of data and materials}

The datasets created and/or analysed during the current study will be available from the corresponding author on reasonable request. There are no security, licensing, or ethical issues related to these data.

\section{Declarations}

\section{Ethics approval and consent to participate}

Ethical approval of this study protocol was obtained by the institutional ethics committee of Guangdong Provincial People's Hospital, Wenzhou Central Hospital and Wenzhou People's Hospital on human research. All procedures involving human participants complied with the ethical standards and with the principle of the Helsinki Declaration of 1964 and its later amendments in 2008. All patients signed an informed consent form before renal biopsy.

\section{Consent for publication}

Not applicable.

\section{Competing interests}

The author(s) declare(s) that they have no competing interests.

\section{Author details}

${ }^{1}$ The Second School of Clinical Medicine, Southern Medical University, Guangzhou 510515, China. ${ }^{2}$ Division of Nephrology, Guangdong Provincial People's Hospital, Guangdong Academy of Medical Sciences, 106 Zhongshan Er Road, Main Building, Room 1436, Guangzhou 510080, Guangdong, China. ${ }^{3}$ Division of Nephrology, Wenzhou Central Hospital, Wenzhou 325000, China. ${ }^{4}$ Division of Renal Pathology, Guangzhou KingMed Diagnostic Laboratory LTD, Guangzhou 510320, China. ${ }^{5}$ School of Medicine, South China University of Technology, Guangzhou 510006, China. 'Division of Nephrology, The First Affiliated Hospital of Wenzhou Medical University, Wenzhou 325000, China. ${ }^{7}$ Shantou University Medical College, Shantou 515041, China. ${ }^{8}$ Division of Nephrology, Wenzhou People's Hospital, Wenzhou 325000, China.
Received: 18 March 2021 Accepted: 3 August 2021 Published online: 18 August 2021

\section{References}

1. Alicic RZ, Rooney MT, Tuttle KR. Diabetic kidney disease: challenges, Progress, and possibilities. Clin J Am Soc Nephrol. 2017;12(12):2032-45.

2. Zhang L, Long J, Jiang $W$, et al. Trends in chronic kidney disease in China. N Engl J Med. 2016;375(9):905-6.

3. Chan JC, Malik V, Jia W, et al. Diabetes in Asia: epidemiology, risk factors, and pathophysiology. JAMA. 2009;301(20):2129-40.

4. Mise $\mathrm{K}$, Hoshino J, Ubara $\mathrm{Y}$, et al. Renal prognosis a long time after renal biopsy on patients with diabetic nephropathy. Nephrol Dial Transplant. 2014:29(1):109-18.

5. Fox CS, Matsushita K, Woodward M, et al. Associations of kidney disease measures with mortality and end-stage renal disease in individuals with and without diabetes: a meta-analysis. Lancet. 2012;380(9854):1662-73.

6. Colombo M, McGurnaghan SJ, Blackbourn LAK, et al. Comparison of serum and urinary biomarker panels with albumin/creatinine ratio in the prediction of renal function decline in type 1 diabetes. Diabetologia. 2020; 63:788-98.

7. Working Group of the International Ig ANN, the Renal Pathology S, Cattran DC, et al. The Oxford classification of IgA nephropathy: rationale, clinicopathological correlations, and classification. Kidney Int. 2009;76(5): 534-45.

8. Working Group of the International Ig ANN, the Renal Pathology S, Roberts IS, et al. The Oxford classification of IgA nephropathy: pathology definitions, correlations, and reproducibility. Kidney Int. 2009;76(5):546-56.

9. Coppo R, Troyanov S, Bellur S, et al. Validation of the Oxford classification of IgA nephropathy in cohorts with different presentations and treatments. Kidney Int. 2014;86(4):828-36.

10. Tanaka S, Ninomiya T, Katafuchi R, et al. Development and validation of a prediction rule using the Oxford classification in IgA nephropathy. Clin J Am Soc Nephrol. 2013;8(12):2082-90.

11. Trimarchi H, Barratt J, Cattran DC, et al. Oxford classification of IgA nephropathy 2016: an update from the IgA nephropathy classification working group. Kidney Int. 2017;91(5):1014-21.

12. Tervaert TW, Mooyaart AL, Amann K, et al. Pathologic classification of diabetic nephropathy. J Am Soc Nephrol. 2010;21(4):556-63.

13. Heaf JG, Lokkegaard H, Larsen S. The relative prognosis of nodular and diffuse diabetic nephropathy. Scand J Urol Nephrol. 2001;35(3):233-8.

14. Mottl AK, Gasim A, Schober FP, et al. Segmental sclerosis and Extracapillary Hypercellularity predict diabetic ESRD. J Am Soc Nephrol. 2018;29(2):694-703

15. Jiang $\mathrm{S}, \mathrm{Yu}$ T, Zhang Z, et al. Prognostic nomogram and score to predict renal survival of patients with biopsy-proven diabetic nephropathy. Diabetes Res Clin Pract. 2019;155:107809.

16. Okada T, Nagao T, Matsumoto H, Nagaoka Y, Wada T, Nakao T. Histological predictors for renal prognosis in diabetic nephropathy in diabetes mellitus type 2 patients with overt proteinuria. Nephrology. 2012;17(1):68-75.

17. Basile D, Donohoe D, Roethe K, Osborn J. Renal ischemic injury results in permanent damage to peritubular capillaries and influences long-term function. Am J Physiol Renal Physiol. 2001;281(5):F887-99.

18. Tanaka T, Nangaku M. Angiogenesis and hypoxia in the kidney. Nat Rev Nephrol. 2013;9(4):211-22.

19. Dimke H, Sparks M, Thomson B, Frische S, Coffman T, Quaggin S. Tubulovascular cross-talk by vascular endothelial growth factor a maintains peritubular microvasculature in kidney. J Am Soc Nephrol. 2015;26(5):1027-38

20. Balachandran VP, Gonen M, Smith JJ, DeMatteo RP. Nomograms in oncology: more than meets the eye. Lancet Oncol. 2015;16(4):E173-80.

21. Vernerey $D$, Huguet $F$, Vienot $A$, et al. Prognostic nomogram and score to predict overall survival in locally advanced untreated pancreatic cancer (PROLAP). Br J Cancer. 2016;115(3):281-9.

22. Wang $H$, Zhang $L$, Liu Z, et al. Predicting medication nonadherence risk in a Chinese inflammatory rheumatic disease population: development and assessment of a new predictive nomogram. Patient Prefer Adhere. 2018;12: 1757-65.

23. Inker $\mathrm{LA}$, Schmid $\mathrm{CH}$, Tighiouart $\mathrm{H}$, et al. Estimating glomerular filtration rate from serum creatinine and cystatin C. N Engl J Med. 2012;367(1):20-9.

24. Levey AS, Inker LA, Matsushita $K$, et al. GFR decline as an end point for clinical trials in CKD: a scientific workshop sponsored by the National Kidney 
Foundation and the US Food and Drug Administration. Am J Kidney Dis. 2014;64(6):821-35.

25. Coresh J, Turin TC, Matsushita K, et al. Decline in estimated glomerular filtration rate and subsequent risk of end-stage renal disease and mortality. JAMA. 2014;311(24):2518-31.

26. Bonsib SM. Renal Anatomy and Histology. In: Jennette JC, D'Agati VD, Olson JL, Silva FG, eds. Heptinstall's Pathology of the Kidney. Vol 1. 7th ed. Amsterdam: Wolters Kluwer Health; 2014. p. 11-157.

27. Lv Y, Wang Z, Li K, et al. Risk stratification based on CLIF consortium acute Decompensation score in patients with child-Pugh B cirrhosis and acute Variceal bleeding. 2020.

28. Camp R, Dolled-Filhart M, Rimm D. X-tile: a new bio-informatics tool for biomarker assessment and outcome-based cut-point optimization. Clin Cancer Res. 2004;10(21):7252-9.

29. Katz MH, Hauck WW. Proportional hazards (cox) regression. J Gen Intern Med. 1993:8(12):702-11.

30. Alba AC, Agoritsas T, Walsh M, et al. Discrimination and calibration of clinical prediction models: Users' guides to the medical literature. JAMA. 2017;318(14):1377-84.

31. Schemper M, Smith TL. A note on quantifying follow-up in studies of failure time. Control Clin Trials. 1996;17(4):343-6.

32. Ishizaki Y, Aizawa A, Ochiai S, Minakawa A, Miyauchi S, et al. A case of lupus vasculopathy presenting favorable renal outcome. CEN Case Rep. 2020;9(1): 74-80.

33. Wu LH, Yu F, Tan Y, Qu Z, Chen MH, Wang SX, et al. Inclusion of renal vascular lesions in the 2003 ISN/RPS system for classifying lupus nephritis improves renal outcome predictions. Kidney Int. 2013;83(4):715-23.

34. Zhang Y, Sun L, Zhou S, Xu Q, Xu Q, Liu D, et al. Intrarenal arterial lesions are associated with higher blood pressure, reduced renal function and poorer renal outcomes in patients with IgA nephropathy. Kidney Blood Press Res. 2018:43(2):639-50.

35. Cai $\mathrm{Q}$, Shi $\mathrm{S}$, Wang $\mathrm{S}$, Ren $\mathrm{Y}$, Hou W, et al. Microangiopathic lesions in IgA nephropathy: A cohort study. Am J Kidney Dis. 2019;74(5):629-39.

36. An Y, Xu F, Le W, Ge Y, Zhou M, Chen H, et al. Renal histologic changes and the outcome in patients with diabetic nephropathy. Nephrol Dial Transplant. 2015;30(2):257-66.

37. Stefan G, Stancu S, Zugravu A, Petre N, et al. Histologic predictors of renal outcome in diabetic nephropathy: Beyond renal pathology society classification. Medicine. 2019;98(27):e16333.

38. Bohle AM, Wehrmann M, Bogenschütz O, Batz C, Müller GA. The pathogenesis of chronic renal failure in diabetic nephropathy. Investigation of 488 cases of diabetic glomerulosclerosis. Pathol Res Pract. 1991;187:251-9.

39. Gambara V, Mecca G, Remuzzi G, Bertani T. Heterogeneous nature of renal lesions in type II diabetes. J Am Soc Nephrol. 1993;3(8):1458-66.

40. Salvatore SP, Reddi AS, Chandran CB, Chevalier JM, et al. Collapsing glomerulopathy superimposed on diabetic nephropathy: insights into etiology of an under-recognized, severe pattern of glomerular injury. Nephrol Dial Transplant. 2014;29(2):392-9.

41. Nyumura I, Honda K, Babazono T, Horita S, et al. Recurrence of diabetic kidney disease in a type 1 diabetic patient after kidney transplantation. Nephrology. 2015;20:90-2.

42. Nadasdy T, Allen C, Zand MS. Zonal distribution of glomerular collapse in renal allografts: possible role of vascular changes. Hum Pathol. 2002;33(4): 437-41.

43. López-Revuelta K, Galdo PP, Stanescu R, Parejo L, et al. Silent diabetic nephropathy. World J Nephrol. 2014;3(1):6-15.

44. Zhao HL, Lai FM, Tong PC, et al. Clinicopathologic characteristics of nodular glomerulosclerosis in Chinese patients with type 2 diabetes. Am J Kidney Dis. 2004;44(6):1039-49.

45. Oh SW, Kim S, Na KY, Chae DW, et al. Clinical implications of pathologic diagnosis and classification for diabetic nephropathy. Diabetes Res Clin Pract. 2012;97(3):418-24.

46. Mise K, Hoshino J, Ueno T, Hazue R, et al. Clinical and pathological predictors of estimated GFR decline in patients with type 2 diabetes and overt proteinuric diabetic nephropathy. Diabetes Metab Res Rev. 2015;31(6): $572-81$

\section{Publisher's Note}

Springer Nature remains neutral with regard to jurisdictional claims in published maps and institutional affiliations.

\section{Ready to submit your research? Choose BMC and benefit from:}

- fast, convenient online submission

- thorough peer review by experienced researchers in your field

- rapid publication on acceptance

- support for research data, including large and complex data types

- gold Open Access which fosters wider collaboration and increased citations

- maximum visibility for your research: over $100 \mathrm{M}$ website views per year

At BMC, research is always in progress.

Learn more biomedcentral.com/submissions 